\title{
ERRORES DE LOS ESTUDIANTES DE OCTAVO GRADO EN EL TRABAJO PRE-ALGEBRAICO
}

\author{
Tulio Rafael Amaya de Armas (UNISUCRE Colombia - UNED España)*
}

\begin{abstract}
Resumen
Esta investigación se basó en la identificación de los errores más comunes que se cometen en el grado octavo al momento de transformar expresiones aritméticas y algebraicas en otras equivalentes. Participaron tres grupos (A, B y C) de educación básica de una institución de carácter oficial. Los estudiantes se reunían por grupos de tal forma que en cada uno hubiera un estudiante monitor, el que se encargaba de explicarles a los compañeros lo que no entendían y a la vez anotaba los errores que les veía cometer; los que se analizaban en reuniones que se hacían en contra-jornada, donde se discutía la postura de cada grupo y se daban indicaciones para futuros encuentros. Resultó muy gratificante el trabajo en grupos en el que los propios estudiantes reportaban los errores de sus compañeros, lo que permitió que los estudiantes que normalmente los cometían, fueran tomando confianza, perdiendo el temor a admitir sus errores y ganando autoridad para cuestionar el trabajo de sus compañeros.
\end{abstract}

\section{Palabras clave}

Pensamiento algebraico - Errores - Remediación - Transición aritmética - Álgebra.

\begin{abstract}
This research focalized in the identification of the most habitual mistakes that eight grade students make when they transform arithmetic and algebraic expressions into other equivalent ones. Three groups ( $A, B$ and $C$ ) of basic education of an official institution participated. The students joined together in groups so that in each of them were a monitor student, who explained to his/her partners what they didn't understand and wrote their mistakes; which were discussed in the other turn of the working day, where the position of each group was analyzed and some indications for next meeting were given. The work together in groups where the proper students reported the
\end{abstract}

- Especialista en Informática Educativa. Universidad Francisco de Paula Santander. Especialista en Biometría. Universidad de Sucre (Colombia). Licenciado en Matemática. Universidad de Sucre. Maestrando en el Master Universitario en Innovación e Investigación en Educación. Universidad Nacional de Educación a Distancia (España). 
mistakes of their partners was very fruitful. It allowed that the students who usually committed, turned self-confident, lost the fear of mistakes and win authority for argue about their partners' work.

\section{Key words}

Algebraic thinking - Mistakes - Remedy - Transition arithmetic-Algebra.

\section{Planteamiento del problema.}

En este trabajo se analizan los errores más comunes que cometen los estudiantes en el proceso de iniciación al álgebra. Errores más relacionados con la manipulación y comprensión de expresiones algebraicas, que con su uso. Y aunque nuestro propósito no fue implementar un proceso de remediación, en un curso de esta naturaleza, tal proceso es inevitable, por lo que resulta muy difícil sólo analizarlos independientes del proceso de remediación, porque a medida que se avanza de un subtema a otro, lo que se dio anteriormente es requerido para lo que sigue, por lo que hay que ir reforzando cada tema.

Los errores de interés en este estudio son de dos tipos: los que son reproducibles y los que no son aislados, (Charnay, 1991), los primeros son aquellos que tienen cierta persistencia y no pueden deberse a la distracción del alumno; los segundos son aquellos que pueden ponerse en relación con otros formando una especie de red o sistema de errores, de tal suerte que existe cierta relación entre unos y otros.

El error se requiere "para afinar la idea individual sobre lo que es falso y lo que es correcto, según una norma dada" (Carrión, 2007, p. 21), es decir, es necesario para que el estudiante pueda distinguir lo correcto de lo incorrecto, lo peligroso es que se quede con él considerándolo como lo correcto. Su análisis proporciona una rica información acerca de cómo se construye el conocimiento matemático; se constituyen en una excelente herramienta para revelar el estado del conocimiento de los alumnos, imprescindible a la hora de realimentar el proceso de enseñanza y aprendizaje con el fin de mejorar los resultados (Del Puerto, Minnaard y Seminara, 2004). La recurrencia de estos errores va creando vacíos conceptuales que a corto o mediano plazo se convierten en serios problemas para el desempeño académico de los estudiantes, llevándolos incluso, hasta la deserción escolar.

Considerando la importancia de los errores que cometen nuestros alumnos, como manifestaciones de las dificultades y los obstáculos, hay que ser conciente de esto y no desfallecer en el intento por hacer converger "la complejidad de los objetos del álgebra, los procesos de pensamiento algebraico, con el desarrollo cognitivo de los alumnos, los métodos de enseñanza y las actitudes afectivas y emocionales hacia el álgebra" (Socas y Palarea, 1997, 17); para que los mismos 
estudiantes noten sus avances con el fin de que no se culpen del aparente fracaso de cualquier actividad de remediación al ver aparecer nuevamente los errores en sus procesos, y sean consientes que "el error es posible en todo proceso de adquisición y consolidación de conocimientos" (Del Puerto, et al, 2004, p. 2), por lo que su análisis y tratamiento puede evitar "consecuencias que, para un país, ocasiona formar profesionales con un aprendizaje en un ambiente de errores", (Carrión, 2007, p. 20). Este autor además considera que para usar un error de manera productiva, es necesario que un individuo pueda comprenderlo, analizarlo, corregirlo y usarlo para desarrollar estrategias de prevención de nuevos errores.

Uno de los resultados más fatales de esta situación lo constituye la actitud que asume el estudiante hacía el álgebra, considerando que "la matemática no es lo mío, esto es para los coquitos de la clase". Este rechazo hacia el álgebra puede estar fundamentada en que "lo que se le pide a los alumnos que realicen en las clases de álgebra no tiene un significado subyacente 0 no están preparados para hacerlo" (Cardona, 2007, p. 4-5). Al respecto el National Council of Teachers of Mathematics (NCTM, 2000, citado por Cardona, 2007), considera que "Ios estudiantes necesitan comprender (del álgebra) sus conceptos, las estructuras y principios que rigen la manipulación de símbolos y cómo pueden usarse éstos para registrar ideas y ampliar su comprensión de las situaciones" (p. 11).

\section{Marco Teórico}

El pensamiento algebraico según Socas (2007), es una línea de estudio e investigación en didáctica de las matemáticas que se ocupa de los fenómenos de enseñanza, aprendizaje y comunicación de los conceptos algebraicos en el sistema educativo y en el medio social, se encuentra inmerso en uno de los grandes dilemas que envuelven el pensamiento matemático: "la cognición es intrínsecamente contextual versus el lenguaje algebraico es intrínsecamente abstracto" Socas (2007, p. 261).

Brousseau (1999) sostiene que el error y el fracaso no tienen el rol simplista que a veces se le quiere hacer representar. El error no es solamente el efecto de la ignorancia, de la incertidumbre, del azar, según se creía en las teorías empiristas o conductistas del aprendizaje; sino que es el efecto de un conocimiento anterior, que tuvo su interés, su éxito, y que ahora se revela falso o simplemente inadaptado. Los errores de este tipo no son imprevisibles, se constituyen en obstáculos. Tanto en el funcionamiento del maestro como en el del alumno, el error es constitutivo del sentido mismo del conocimiento adquirido.

Según Charnay, (1991) los errores se consideran significativos, y poseen las características de ser "reproducibles", cuando se manifiesta una cierta persistencia, por lo tanto no es considerado al azar, o por distracción. Además sostiene que estos hechos no son aislados pueden ponerse en relación con otros. 
Por lo que los errores así conformados propician la creación de patrones de comportamiento equivocados en la ejecución de las actividades planteadas.

Ruano, Socas y Palarea (2008), afirman que el aprendizaje del álgebra genera muchas dificultades a los alumnos, además que estas dificultades son de naturaleza diferente, y tienen que ver con la complejidad de los objetos del álgebra. Aluden a que los errores aparecen en el trabajo de los alumnos sobre todo, cuando se enfrentan a conocimientos novedosos que los obliga a hacer una revisión o reestructuración de lo que ya saben.

El error según Del Puerto, et al (2004), "es considerado parte inseparable del proceso de aprendizaje. Los investigadores en educación matemática sugieren diagnosticar y tratar seriamente los errores de los alumnos, discutir con ellos sus concepciones erróneas, y presentarles luego situaciones matemáticas que les permitan reajustar sus ideas" (p. 2), para ir preparando el camino hacia temas de mayor complejidad donde se requiera la presencia de conceptos básicos que se hayan afianzado previamente. Este proceso de reajuste de ideas es lo que Charnay (1991) llama remediación y lo define como "todo acto de enseñanza cuyo objetivo es permitir que el alumno se apropie de los conocimientos, después de que una primera enseñanza no le ha permitido hacerlo de forma perfecta" (p. 2).

Desde 1938, Bachelard ya consideraba que los entorpecimientos y confusiones, que causan estancamientos y retrocesos en el proceso del conocimiento, provienen de una tendencia a la inercia, a la que da el nombre de obstáculo; que se conoce en contra de un conocimiento anterior, destruyendo conocimientos mal hechos, superando lo que en la mente hace de obstáculo y, por lo tanto, los errores son constitutivos del propio acto de conocer. Bajo este punto de vista pretender que los estudiantes no cometan errores sería pretender que dejaran de aprender.

Albert (1997), hace una clasificación de los obstáculos, en el sentido propuesto por Brousseau, donde plantea los orígenes según la procedencia de éstos; los hay de origen ontogénicos, que sobrevienen de las limitaciones del sujeto en un momento de su evolución, los de origen didáctico, relacionados con el modo como se enseñan los conocimientos de acuerdo a un modelo educativo especifico, es decir, son los vinculados con la metodología utilizada por el profesor en el proceso de aprendizaje, y los de origen epistemológico, son dificultades intrínsecas de los conocimientos, o sea, relacionados con la dificultad propia del concepto que se aprende y que además pueden ser rastreados a lo largo de la historia de la matemática, en la génesis misma de los conceptos. En esta perspectiva la responsabilidad del error no es atribuida exclusivamente al alumno, caben otras posibilidades, pero en todos los casos se destaca el carácter de resistentes que presentan estos obstáculos, y es necesaria su identificación, para luego alcanzar los nuevos conocimientos a partir de su superación. 
Según Arce, Torres Ramírez, Valoyes, Malagón y Arboleda (2005), una creencia generalizada entre los profesores de matemáticas es considerar que el paso de la aritmética al álgebra es automático. Estos autores también consideran, que para la construcción del conocimiento algebraico, además de la intervención didáctica, se necesitan cambios conceptuales, en lo simbólico relacionados con la noción de igualdad, la interpretación de la letra y las convenciones y transformaciones para resolver ecuaciones entre otras. La transición de la aritmética al álgebra es considerada como el conjunto de "cambios en los tipos de convenciones usadas en la aritmética e interpretaciones de la letra en contextos matemáticos" (Rojas, Rodríguez, Romero, Castillo y Mora, 1999 , p. 9), además, del reconocimiento y uso de las estructuras, el cambio de convenciones respecto a la concatenación de los símbolos que se usan en aritmética y en álgebra respectivamente; por ejemplo en aritmética el símbolo 36 significa $30+6$, esto es, tres decenas y seis unidades, mientras en álgebra escribir $3 x$ representa la multiplicación del número 3 con $x$. Como el estudiante viene acostumbrado a esta convención, le cuesta aceptar otro significado para una concatenación muy similar de estos símbolos.

Carrión (2007), clasifica los errores cometidos en el proceso pre-algebraico en tres tipos: errores de entrada, de operación y de escritura:

- Los errores de entrada, son errores en la lectura del texto o de visión, en éstos los estudiantes aunque realizan los cálculos en forma correcta, operan una expresión diferente a la que se les propone, es decir, eligen el proceso correcto, pero presentan errores en su proceso de solución, cambiando por ejemplo los términos de las expresiones o inventando términos que no están originalmente en las expresiones; estos errores por lo general conducen a resultados incorrectos.

- En los errores de operación los estudiantes distorsionan el proceso de obtener el resultado de cada operación realizada en forma independiente; por ejemplo, cambiando los signos, u omitiéndolos, multiplicando la base por el exponente al ejecutar una potenciación, sumando los numeradores y también los denominadores al sumar fraccionarios, entre otros.

- Y los errores de escritura, son errores de una salida de etapa, no de salida del proceso completo, se presentan al comunicar el procedimiento de transformación de la expresión, aunque se escojan las operaciones adecuadas y estas se realicen correctamente; en los errores de este tipo, el estudiante realiza los cálculos secuencialmente sin cometer errores en la ejecución de las operaciones y es muy común que se obtengan resultados correctos. 


\section{Metodología de trabajo}

En este trabajo se hace el análisis de los errores con base a la transformación de una expresión aritmética en otra equivalente. Esto se hace en el desarrollo ordinario de un curso de octavo grado, con los temas habituales que según los Estándares Básicos de Competencias en Matemáticas que rigen en Colombia, deben desarrollarse en tal grado.

El trabajo se realizó en el segundo semestre académico del año 2009. La muestra la conformaron 91 estudiantes del grado octavo de educación básica secundaria, repartidos en tres grupos A (30), B (29) y C (32), con edades entre 13 y 14 años, provenientes de los estratos socioeconómicos más bajos (1 y 2), de la Institución Educativa Madre Amalia del municipio de Sincelejo, Colombia, todos de la jornada matinal.

Después de cada evaluación el docente seleccionaba algunos exámenes para ser analizados en grupos en la siguiente clase, previo a la actividad de recuperación. En cada salón los estudiantes se reunían por grupos de tres o cuatro estudiantes, cada uno con un monitor seleccionado por el docente (uno de los estudiantes más avanzados en la clase de matemáticas), quien se encargaba de anotar los errores y de explicarles a los compañeros lo que ellos no entendían. En contra-jornadas se hacían reuniones con los monitores, entre los que se formaban grupos, con miembros de cada uno de los salones; cada grupo designaba un relator quien debía exponer ante los demás los errores que habían visto cometer a sus compañeros en la clase, se discutía la postura de cada grupo y con base en esto se acordaba lo que se debía hacer en el próximo encuentro. Luego se escogía entre todas las expresiones tipo analizadas, una para trabajar en profundidad y se iban dejando en el registro. Algunas de estas expresiones son las analizadas en este escrito.

El papel del profesor en general consistió en acompañar a los estudiantes, como moderador en las situaciones de conflicto social, evitando que se dieran situaciones de autoridad por cuenta del rendimiento académico "yo tengo la razón porque soy mejor estudiante que tú", buscando que la autoridad a un estudiante se la diera más la razón que los buenos promedios académicos. De esta forma se veían obligados a explicar el porqué de las cosas, cuando se presentaban conflictos académicos donde cada uno defendía su resultado. Y cuando se encontró una dificultad generalizada en todos los grupos, se hizo una intervención para todos, casi siempre consistente en preguntas o comentarios que los llevaran a reflexionar o cuestionar lo que habían hecho. Además, el profesor, observando el trabajo de los grupos, llevaba un pre-inventario de los errores que los veía discutir. En las reuniones contra-jornada se profundizaba en los temas y se daban orientaciones para los siguientes encuentros en los salones.

El trabajo se realizó en varios momentos:

- Discusiones preliminares para conceptualizar el problema de investigación y acordar la metodología de trabajo. 
- Conformación de grupos, análisis y discusión de algunas bases teóricas sobre análisis de errores.

- Trabajo en grupos en las clases para recoger la información e intentar minimizar los errores encontrados.

- Análisis y discusión por grupos de monitores, de los errores encontrados en la clase.

- Socialización y discusión en plenaria de los errores encontrados por los monitores e implementación de nuevas estrategias.

A los estudiantes de la muestra se les pidió que expresaran por escrito el proceso y el resultado al realizar los cálculos en las siguientes expresiones:

1. $25 \times 354-84 \times 6^{5}+8 \times 21$

2. $\left(\frac{2}{3}\right)^{4} \times\left(\frac{5}{4}\right)^{3}$

3. $\left(\frac{2}{3}\right)^{3}+\left(\frac{5}{4}\right)^{2}$

Para el análisis hubo que entrevistar a algunos estudiantes para aclarar algunas respuestas que resultaron complicadas de interpretar, con lo que se ampliaron o complementaron algunas respuestas.

\section{Resultados}

A continuación se presenta el porcentaje de estudiantes que presentó dificultad haciendo la transformación en cada una de las expresiones que se les propusieron, y se analizan los errores cometidos por algunos de ellos, de acuerdo al tipo de error cometido, expresión por expresión. Por simplicidad sólo se analizan tres expresiones.

\section{Expresión 1. $25 \times 354-84 \times 6^{5}+8 \times 21$}

En relación con la expresión 1, el 50,55\% (46) de los estudiantes cometió algún error al hacer la transformación de esta expresión en otra equivalente. Así mismo, fue amplia la variedad de errores que se presentaron. A continuación se muestran algunos de estos errores, presentados por los estudiantes y algunos de sus manuscritos al tratar de realizar los cálculos en cada una de las expresiones propuestas.

\section{Ejemplo 1.}

Este estudiante comete un par de errores de entrada, como se puede ver en su manuscrito, además, omitió realizar la última operación. Puede apreciarse que los cálculos realizados los ejecutó correctamente y por separado; los errores 
están en la combinación de términos, ya que las operaciones realizadas no son las propuestas en el ejercicio.

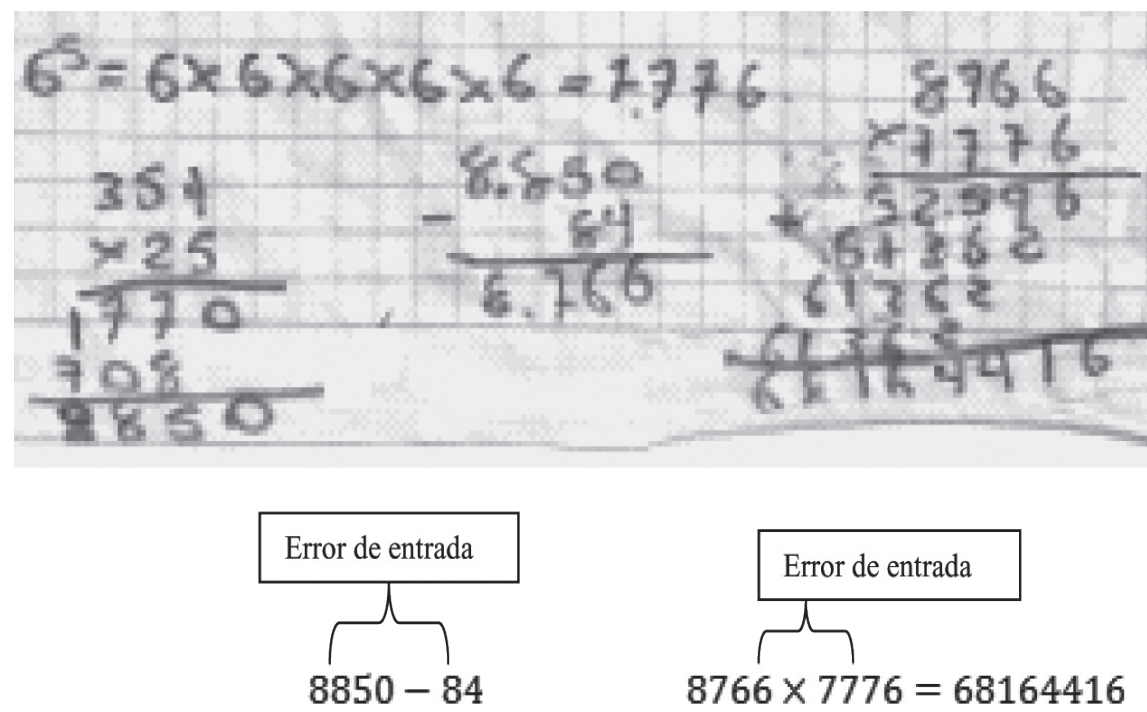

En la forma de operar de este estudiante, persiste una práctica verbal de lo escrito, por cuanto no respeta las reglas de la aritmética. Quizás por desconocimiento de que la lengua matemática escrita difiere de la lengua matemática hablada (Carrión, 2007).

\section{Ejemplo 2.}

Este estudiante agrupa los términos con igual signo y realiza cada operación por separado. Al realizar la operación con los términos que tienen signo negativo, omite el signo, desarrolla la potencia y posteriormente comete un error de escritura al continuar secuencialmente con los cálculos sin separarlos. Al final vuelve y considera el signo negativo. Cuando ya tiene las cantidades positivas y negativas correspondientes procede a hacer la adición y comete un error de operación, al realizar el cálculo en forma incorrecta.

Cabe anotar que fue uno de los pocos estudiantes que en este ejercicio obtuvo un resultado negativo. Al indagársele sobre las razones por las que no utilizó el signo negativo en el proceso, respondió que él en la realidad encuentra por ejemplo, dos o cinco panes, no menos dos o menos cinco panes, pero que colocó el signo al final porque sabía que iba en el ejercicio y lo debía utilizar por cumplir con éste. Lo expresado por este alumno, es un ejemplo de que "las concepciones de un sujeto sobre un concepto matemático designan el modo en 
el que ese sujeto comprende y utiliza el concepto" (Sierra, González y López, 2000 , p. 72), al asociar el número a su cardinalidad, relacionándolos sólo con cantidades, lo que podría estar impidiéndole concebir los números negativos, convirtiéndose esto en un obstáculo en el sentido de Albert (1997); algo que, en cierto sentido, puede considerarse normal, teniendo en cuenta la sorprendente lentitud del proceso histórico de construcción del concepto de número negativo (Cid y Bolea, 2007); concepto que parece no haber sido concebido por el alumno por no poderlo relacionar con algo conocido (Brousseau, 1999).

El comportamiento del grupo en general ante esta situación deja ver un patrón comportamental frente al trabajo con números negativos, que da luces para una futura intervención, dada la necesidad de la apropiación de este concepto, estructurante de la disciplina, y del proceso de aprendizaje de saberes matemáticos del niño, y teniendo en cuenta que "el tratamiento adecuado de errores puede contribuir a mejorar el proceso de aprendizaje" (Carrión, 2007, p. 20).
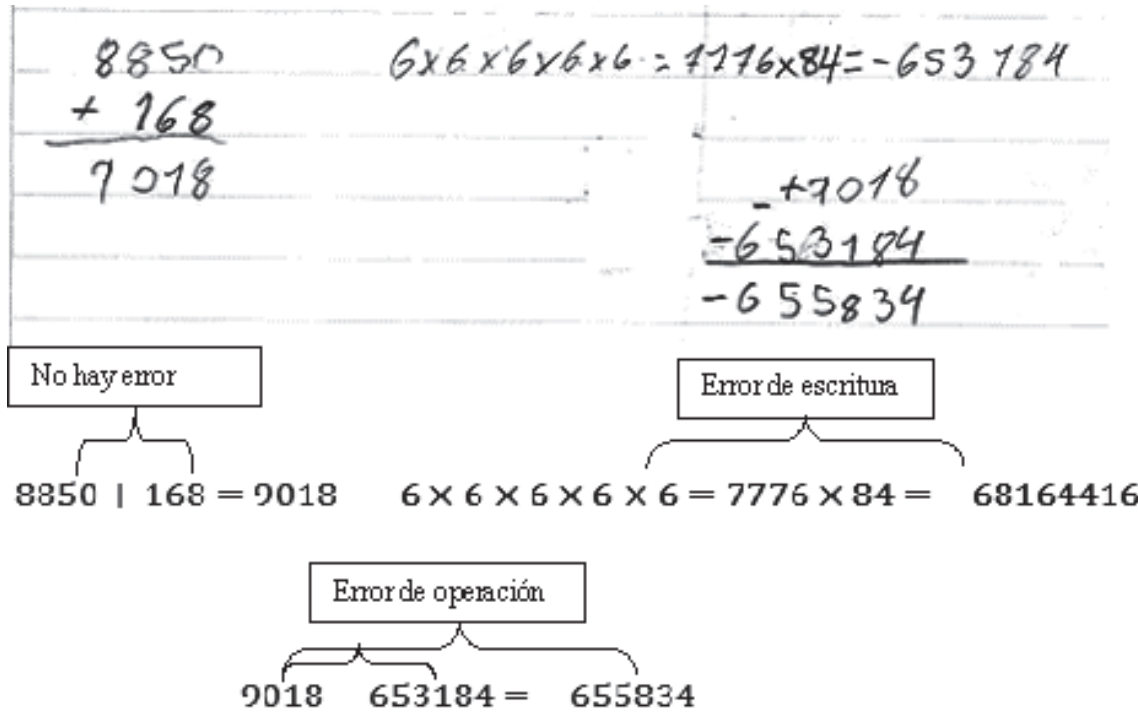

\section{Ejemplo 3.}

Este estudiante al igual que el anterior, realizó cada operación por separado. Cometiendo un error de operación al cambiar el minuendo con el sustraendo, persistiendo la idea de que al restar dos números, el minuendo debe ser mayor que el sustraendo. El segundo error se dio como consecuencia del primero, formándose lo que Charnay (1991) llama red o cadena de errores, ya que la operación realizada fue correcta, pero no corresponde a lo planteado en el ejercicio. 

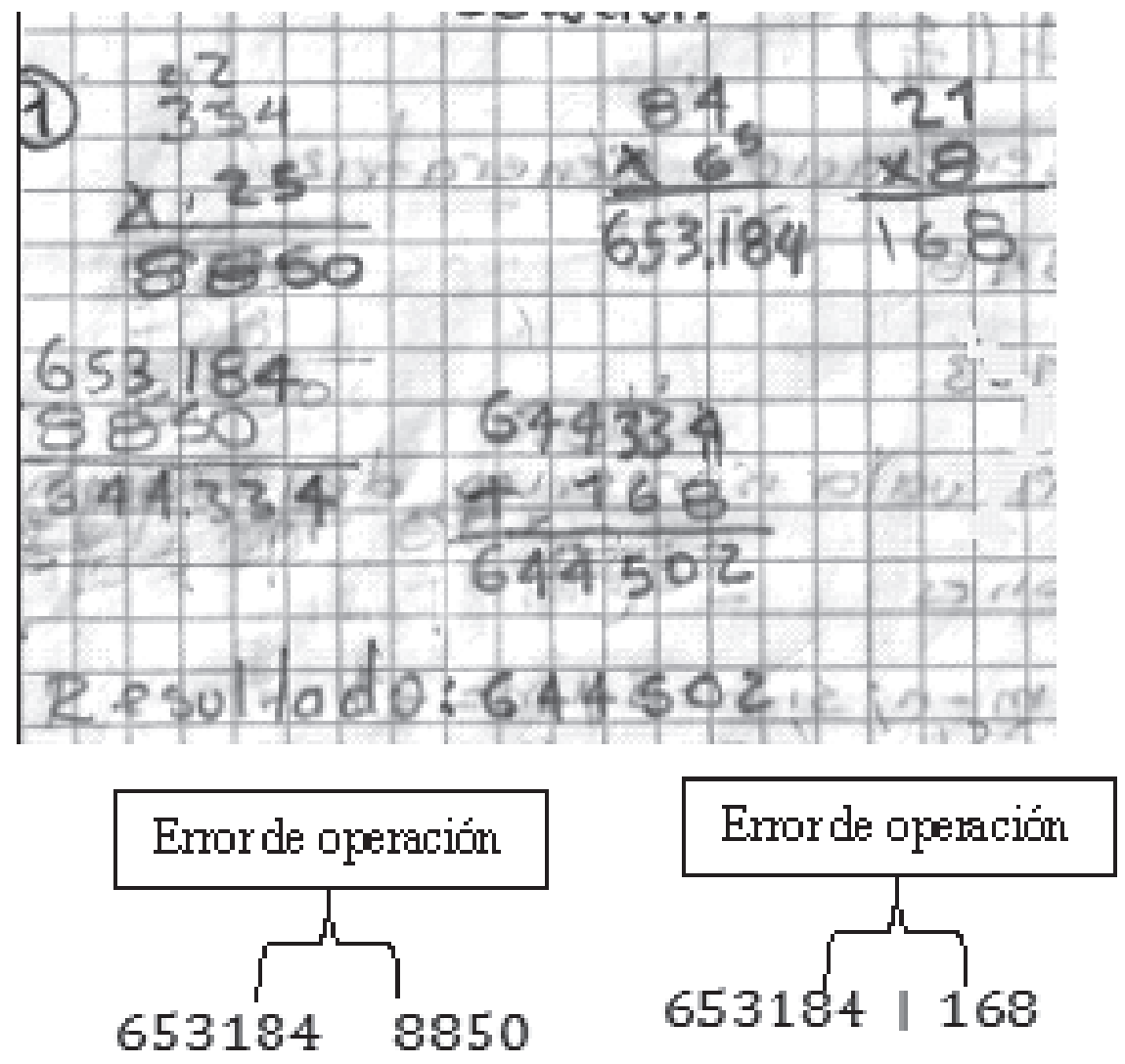

\section{Ejemplo 4.}

Este fue uno de los errores más comunes entre los estudiantes de la muestra. Cometió sucesivos errores de entrada que alteraron ostensiblemente el resultado. En cada paso viola las reglas de la aritmética, al no realizar las operaciones en el orden de prioridad convencionalmente establecido en la disciplina. Paralelamente a los errores de entrada, el estudiante cometió una serie de errores de escritura. Al final del manuscrito del estudiante, se muestra el algoritmo que este siguió para obtener su respuesta. Este tipo de errores combinados fue uno de los más afectados por el trabajo en grupo de los estudiantes, ya que fueron fácilmente detectados, y ayudados a corregir, por los estudiantes líderes, pero a su vez fue uno de los más recurrentes. 

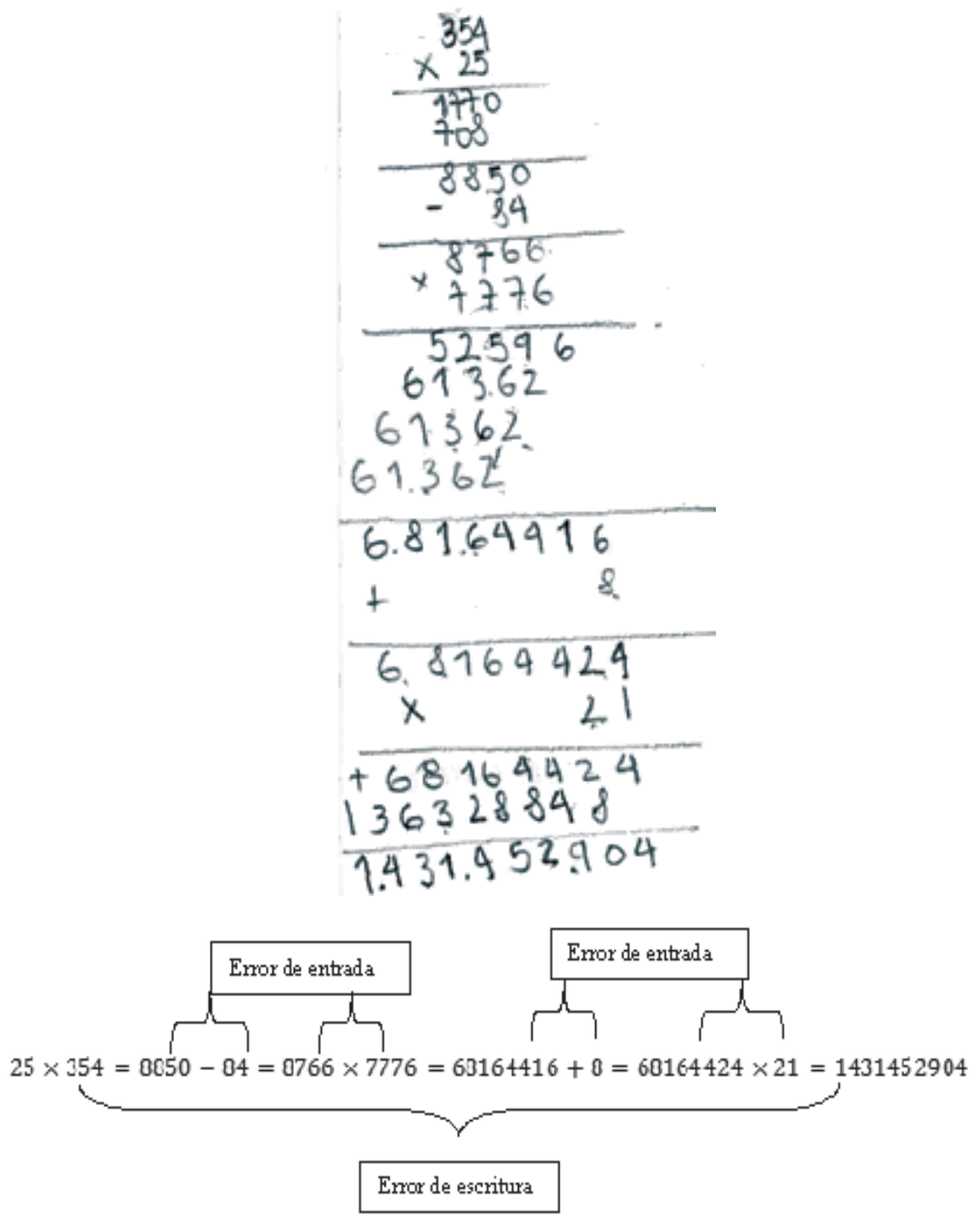

\section{Ejemplo 5.}

En este caso el estudiante comete un par de errores de escritura, ejecutando correctamente los cálculos, pero siguiendo una secuencia inapropiada de las operaciones, obteniendo finalmente un resultado correcto. Este tipo de errores 
fue muy difícil de detectar por los estudiantes líderes de los grupos, quienes no los consideraban un error, ya que, como el resultado final es correcto, no encontraban justificación para decirles a sus compañeros que se trataba de un error, y aunque algunos los detectaron, necesitaban de la confirmación del docente para afirmar que era un error. Este error fue causado por la transcripción fiel de los pasos al ingresar la expresión en la calculadora, hecho que tomaba el estudiante como base para justificar que esto no era un error.

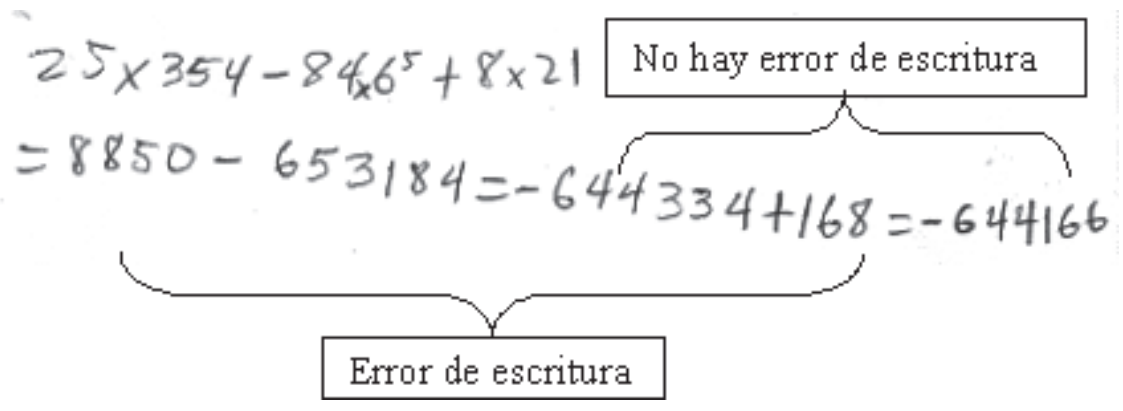

Expresión 2. $\left(\frac{2}{5}\right)^{4} \times\left(\frac{5}{4}\right)^{3}$

En relación con la expresión 2, el 73,62\% (67) de los estudiantes cometió algún error al hacer la transformación de esta expresión en otra equivalente. Algunos de los errores que se presentaron se muestran en los ejemplos del 6 al 9.

\section{Ejemplo 6.}

Aquí el estudiante ejecuta correctamente las potencias en ambos fraccionarios, sin embargo al realizar la multiplicación de los fraccionarios resultantes, comete un error de operación cuando en lugar de realizar la multiplicación que tiene planteada, lo que hace es multiplicar en cruz los términos de los fraccionarios y luego los denominadores, como si fuera una suma. En el manuscrito de este estudiante se puede apreciar que este error no es el efecto de su ignorancia, sino de un conocimiento anterior, que le funcionó perfectamente en la suma y que ahora quiso que le funcionara de igual manera en la multiplicación con fraccionarios, pero que en este caso se revela como falso (Brousseau, 1999). 


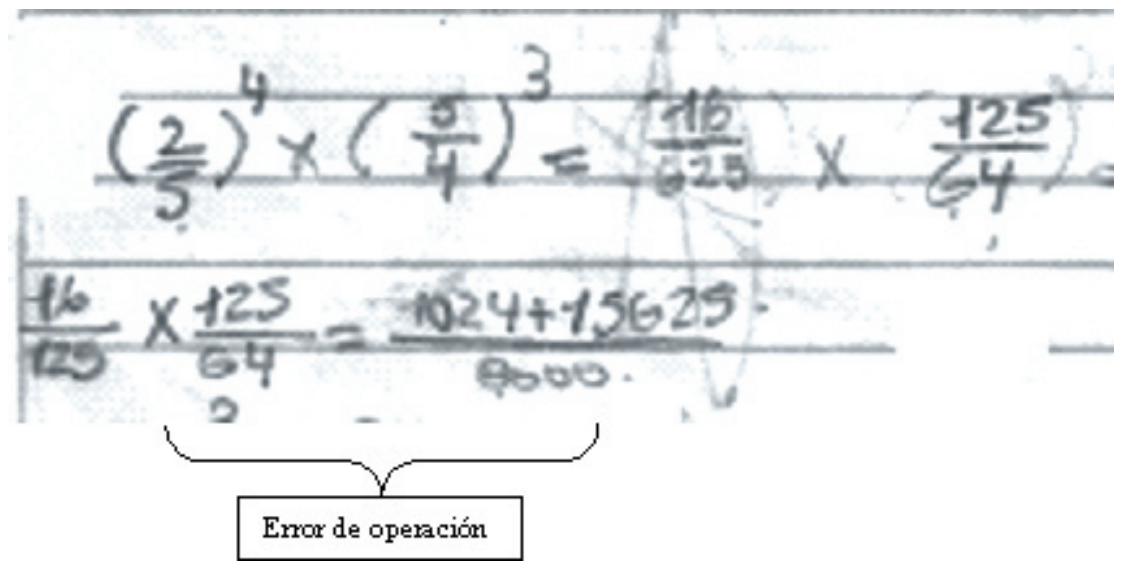

\section{Ejemplo 7.}

Aquí el estudiante parte de sendos errores de operación al considerar $\frac{2^{4}}{5^{4}}=\frac{2 \times 4}{5 \times 4}=\frac{8}{20} \frac{2^{4}}{5^{4}}=\frac{2 \times \mathbf{4}}{5 \times 4}=\frac{\mathbf{8}}{20}$ y $\frac{5^{3}}{4^{3}}=\frac{5 \times 3}{4 \times 3}=\frac{15}{12}$ pretendiendo que la potenciación funcione como la multiplicación. En el tercer término comete otro error de operación al multiplicar en cruz el numerador del primer fraccionario con el denominador del segundo y el denominador del primero con el numerador del segundo, como en una suma de fraccionarios. $Y$ finalmente en lugar de ejecutar la multiplicación que tenía planteada, lo que hace es la suma de los factores que aparecen en el numerador de la fracción. Los errores en este ejemplo muestran la tipología de los obstáculos en el sentido planteado por Albert (1997) y al operar con fraccionarios, ejecutar las potencias como multiplicaciones y la multiplicación como si se tratara de una suma.

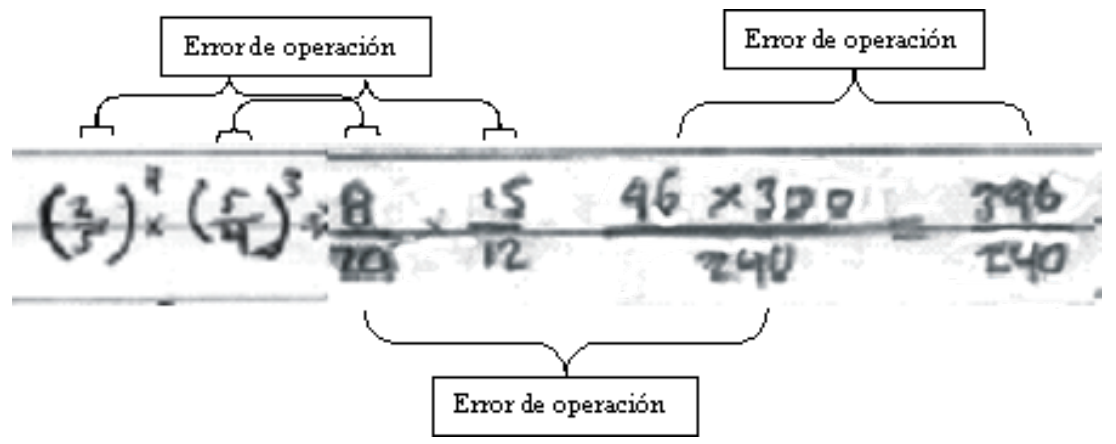




\section{Ejemplo 8.}

En este caso se ejecutan correctamente las potencias, se plantea la multiplicación de los fraccionarios resultantes, pero al realizarla se comete un error de operación, al multiplicar en cruz los términos de los fraccionarios como si fuera una suma. Finalmente se ejecuta la multiplicación que planteó.

\section{$2 \times 2 \times 2 \times 2=16 \quad 5 \times 5 \times 5=115 \quad \frac{16}{81} \times 125=1024 \times 10125-103620$

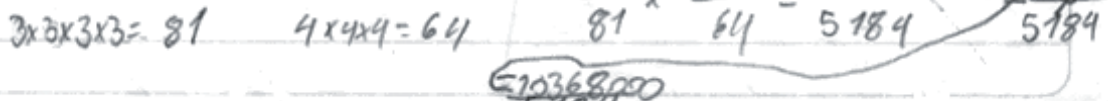

Los errores de operación como los de los ejemplos 6 al 8, fueron una constante en todo el proceso; se hacían actividades para remediarlos, según el tipo (Charnay, 1991), y volvían a aparecer de la misma forma o diferente, pero siempre aparecían. Este tipo de error fue el más persistente, y siempre los mismos estudiantes tendían a cometer los mismos errores.

\section{Ejemplo 9.}

En este ejemplo el estudiante ejecuta correctamente las potencias, pero comete errores de escritura y finalmente obtiene un resultado correcto, que no lo es por ser producto de un proceso inadecuado (Carrión, 2007).

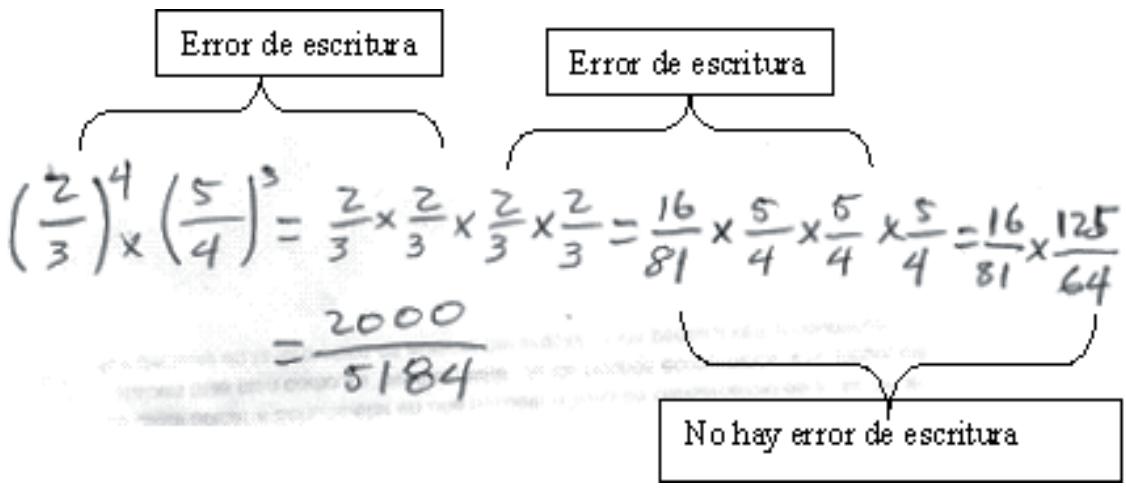

\section{Expresión 3. $\left(\frac{2}{3}\right)^{3}+\left(\frac{5}{4}\right)^{2}\left(\frac{2}{3}\right)^{3}+\left(\frac{5}{4}\right)^{2}$}

En relación con la expresión 3, el 46,15\% (42) de los estudiantes cometió algún error al hacer la transformación de esta expresión en otra 
equivalente y al igual que en las anteriores, fueron diversos los errores que se presentaron. A continuación mostramos algunos ejemplos.

\section{Ejemplo10.}

Aquí el estudiante comienza con un error de escritura y termina los cálculos con un error de operación. Puede apreciarse que en el desarrollo de las potencias no comete errores, realiza cada potencia por separado, pero al iniciar la suma comete un error de escritura, presentando los resultados por etapas (Carrión, 2007).
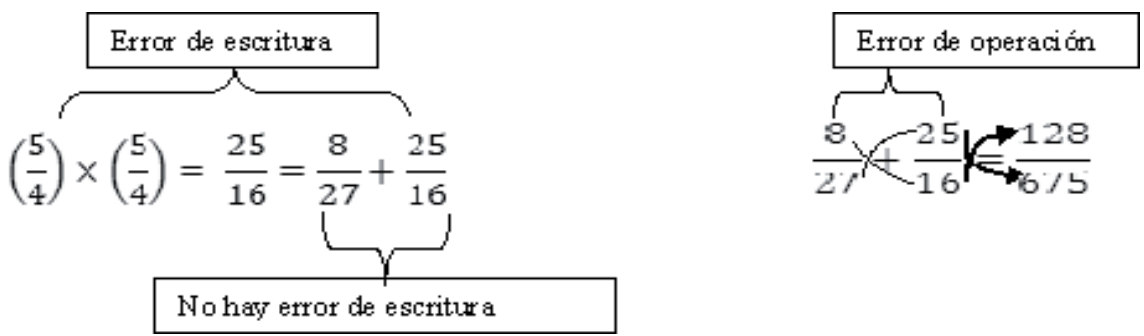

Al realizar la suma de fraccionarios comete un error de operación, queriendo hacer funcionar la suma como la división de estos números, colocando la primera multiplicación en el numerador y la segunda en el denominador.

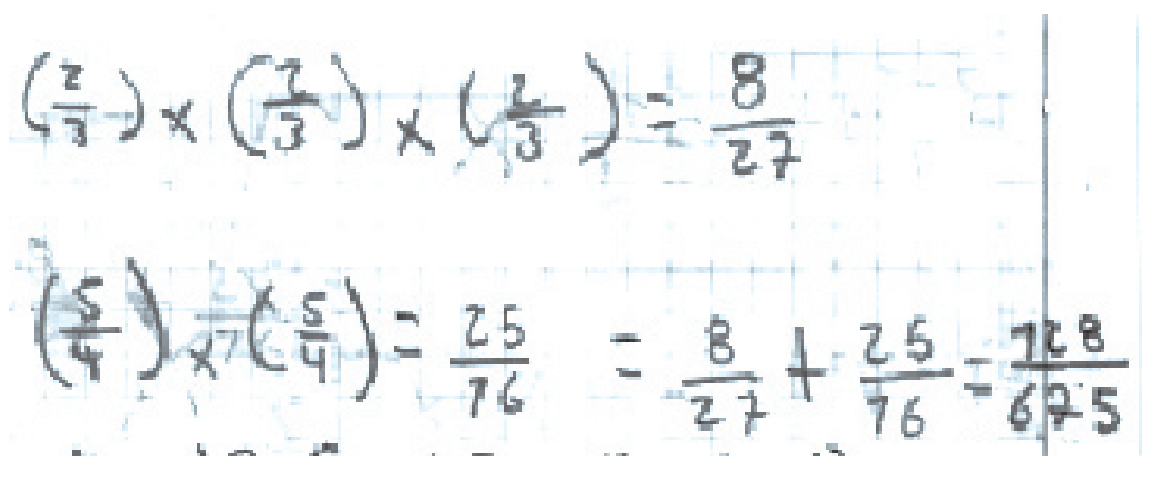

\section{Ejemplo 11.}

Aquí el estudiante cambió la expresión por,

$$
\left(\frac{2}{3}\right)^{3}+\left(\frac{5}{4}\right)^{2} \frac{2 \times 3}{3 \times 3}+\frac{5 \times 2}{4 \times 2}
$$

cometiendo así un par de errores de operación, los cálculos siguientes los realiza correctamente. En este ejemplo se muestra también la presencia de 
obstáculos en el sentido de Brousseau. El estudiante multiplicó el exponente por el numerador y el denominador en cada fracción, teniendo en cuenta que el exponente afecta tanto al numerador como al denominador, pero sin respetar las reglas de la aritmética al expresar las potencias como producto de factores iguales. Observándose que el conocimiento funcional en un contexto es disfuncional en otro más amplio, en el cual se torna más bien en un obstáculo epistemológico Barrante (2006); esto es lo que Brousseau (1999) denomina dominio de validez de un obstáculo.

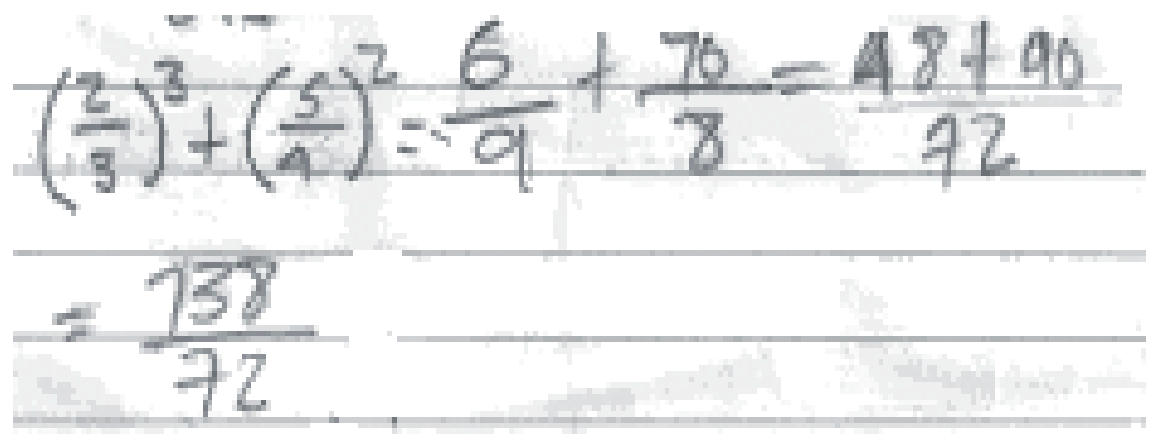

\section{Ejemplo 12.}

En este caso el estudiante comete un error de escritura en la secuencialidad de la ejecución de las operaciones, pero llegando a la respuesta esperada.

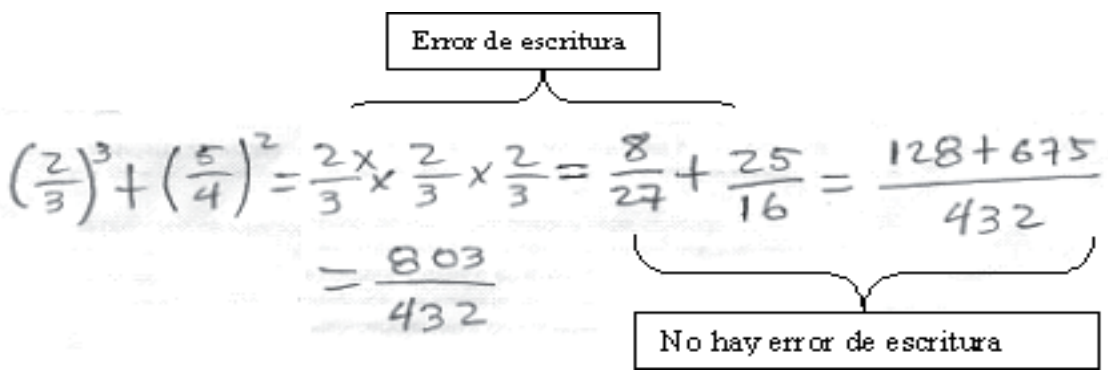

Este tipo de errores fueron los más sistémicos: se dieron como en serie, unos como consecuencia de los otros, lo que Perrot (1989, citado por Charnay, 1991) Ilama coherencia entre errores. Coincidimos con Carrión (2007), que aunque los estudiantes eligieran el algoritmo correcto, parte de ellos presentaron errores en el proceso de resolución.

En la siguiente tabla se presenta un resumen sintético de los tipos de errores cometidos por los estudiantes. 


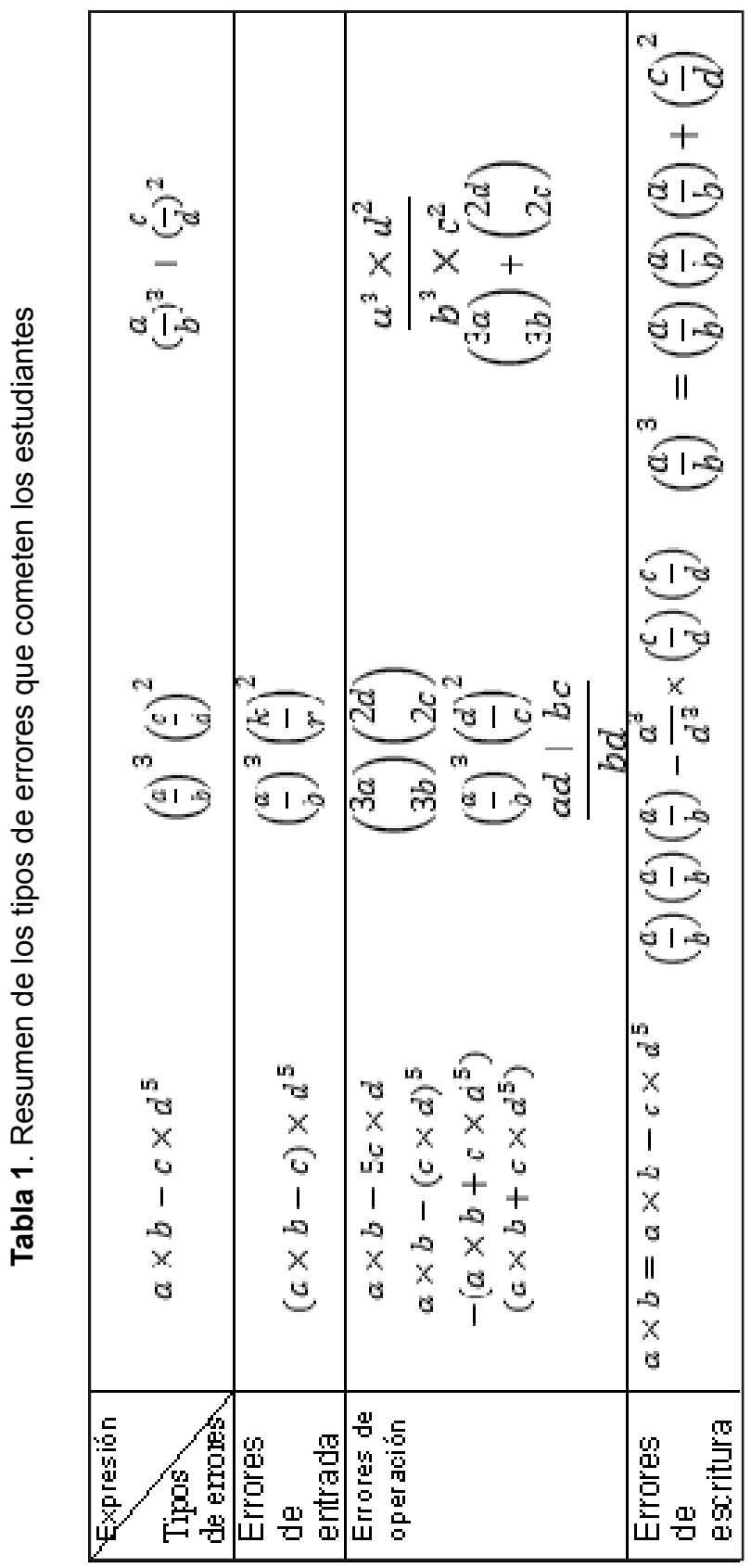




\section{Reflexiones finales}

Luego de este análisis de los errores que cometen los estudiantes, como indicio de los obstáculos en el sentido propuesto por Albert (1997), surgen algunos interrogantes sobre el proceso de intervención, a la vista de los resultados obtenidos. ¿Se debería hacer un trabajo inicialmente en el contexto donde el dominio es válido?, ¿intentar franquear el conocimiento que obstaculiza? 0 ¿trabajar directamente sobre los errores?, para, sino eliminarlos, por lo menos, sí minimizarlos. ¿A cuáles prácticas sociales están asociados estos errores?, ¿cuál es su uso en la comunidad donde se desarrollan estos estudiantes? ¿Cuáles concepciones debe sustituir el estudiante para superar sus obstáculos? ¿Qué cuidado se debe tener, para que las concepciones que se sustituyan no se conviertan en un obstáculo en otro contexto?

De todas formas, a partir de los resultados que se exponen y tomando en cuenta la realidad comentada, surge la necesidad de hacer un trabajo con profesores y alumnos, y demás actores que incidan en la formación de los escolares; con la participación de todos los implicados en la tarea educativa (Carrión, 2007).

Resultó muy interesante el trabajo de los estudiantes líderes de los grupos, quienes con mucho entusiasmo y autoridad ayudaron a sus compañeros a identificar sus errores y, a su manera, les explicaron cómo corregirlos. Este hecho permite, visualizar avances en el proceso de enseñanza y aprendizaje de los estudiantes, quienes ven en su labor una oportunidad de mejoramiento personal, ya que cuando tuvieron que explicar algo de lo que tenían dudas, les tocaba consultar para poder ayudar a sus compañeros.

\section{Conclusiones}

Los errores de entrada se debieron a la prioridad que los estudiantes dan a las operaciones, realizando primero sumas o restas que multiplicaciones; por operar con números que no estaban en los ejercicios, en general, por no respetar las reglas de la aritmética.

Los errores de operación se produjeron por cambios en los signos de las operaciones, por invertir los factores en un fraccionario, uso inadecuado de las leyes de la potenciación, omisión de alguna operación o de un número y cambio de operaciones, como potenciación por multiplicación o multiplicación por división o por suma.

Los errores de escritura fueron los menos admitidos por los estudiantes, los cometían y como casi siempre obtenían la respuesta correcta, sostenían que eso no podía ser un error porque el resultado estaba bien. Los mismos monitores no los veían como error. Estos errores se debieron más que todo a formas inadecuadas en la sintaxis, al encadenar varios signos de igualdad operación tras operación sin alterar el orden ni la prioridad de las operaciones y en la mayoría de los casos realizando cálculos correctos. 
El hecho de analizar las producciones de los estudiantes, permite conocer sus errores más frecuentes y plantear actividades de remediación (Vergnaud, 1991, citado por Carrión, 2007) en función de estos errores, lo que podría llevar a los profesores a realizar algunas modificaciones en el currículo, basado en una realidad muy propia, además, puede permitirle al profesor "diseñar problemas de investigación adaptados al nivel de los alumnos" (Carrión, 2007, p. 55).

Uno de los aspectos más importante del trabajo lo constituyó el trabajo en grupo de los estudiantes, este trabajo entre pares permitió un acercamiento de tú a tú, el mismo hecho de que fueran los propios compañeros los que los hicieran caer en cuenta de los errores cometidos, permitió que los estudiantes que cometían errores con mucha frecuencia, perdieran el temor de admitir cuando tenían resultados errados, lo que les fue dando autoridad para reconocer sus errores y también los de los demás.

\section{Referencias bibliográficas}

- Albert, A. (1997). Introducción a la epistemología. En Serie Antologías (pp. 1-28). México. Área de Educación Superior Departamento de Matemática Educativa, Centro de investigación y de estudios avanzados de IPN.

- $\quad$ Arce, J. Torres, L. Ramírez, M. Valoyes, L. Malagón, M. y Arboleda, L. (2005). Iniciación al álgebra escolar: situaciones funcionales, de generalización y modelación. Universidad del Valle. Conciencias. Cali. Colombia.

- Barrante, H. (2006). Los Obstáculos epistemológicos. Cuadernos de Investigación y formación en Educación Matemática. http://cimm.ucr.ac.cr/cuadernos/cuaderno2/ Cuadernos\%202\%20c\%204.pdf.

- Brousseau, G. (1999). Los Obstáculos Epistemológicos y los Problemas en Matemáticas. Traducido por Hernández y Villalba del original: Brousseau, G. (1983). Les obstacles épistémologiques et les problèmes en mathématiques. Recherches en Didactique des Mathématiques, 4(2), 165-198.

- Cardona, M. (2007). Desarrollando el pensamiento algebraico en alumnos de octavo grado del Centro de investigación e innovación educativa de la Universidad Pedagógica Nacional Francisco Morazán a través de la resolución de problemas. Tesis doctoral no publicada. Universidad Pedagógica Nacional Francisco Morazán.

- Carrión, V. (2007). "Análisis de errores de estudiantes y profesores en expresiones combinadas con números naturales." Unión, Revista Iberoamericana de Educación Matemática, $\mathrm{N}^{\circ} 11$, pp. 19-57.

- $\quad$ Cid, E. y Bolea, P. (2007). Diseño de un modelo epistemológico de referencia para introducir los números negativos en un entorno algebraico. http://www4.ujaen.es/ aestepa/TAD_II/ Comunicaciones_TAD_II/11\%20-\%20Cid\&Bolea\%20TAD\%202.pdf

- Charnay, R. (1991). Del análisis de los errores en matemáticas a los dispositivos de remediación: algunas pistas Equipo de Investigación en didáctica de la Matemática INRP. Michel Mante del IREM de Lyon. En: Grand N, N 48, pp. 37-64

- $\quad$ Del Puerto, S. Minnaard, C. y Seminara, S. (2004). "Análisis de los errores: una valiosa fuente de información acerca del aprendizaje de las Matemáticas." En Revista Iberoamericana de Educación, 38(4), 1-13. 
- Rojas, P. Rodríguez, J. Romero, J. Castillo, E. y Mora, L. (1999). La transición aritmética-álgebra. Universidad Distrital Francisco José de Caldas. Santa Fe de Bogotá, Colombia.

- $\quad$ Ruano, R. Socas, M. y Palarea, M. (2008). Análisis y clasificación de errores cometidos por alumnos de secundaria en los procesos de sustitución formal, generalización y modelización en álgebra. PNA 2(2), 61-74.

- Sierra, M. González, M. López, C. (2000). "Concepciones de los alumnos de bachillerato y curso de orientación universitaria sobre límite funcional y continuidad." En Revista Latinoamericana de Investigación en Matemática Educativa, 3(1), 71-85. Comité Latinoamericano de Matemática Educativa. México.

- $\quad$ Socas, M. y Palarea, M. (1997). "Las fuentes de significado, los sistemas de representación y errores en el álgebra escolar." En Uno. Revista de Didáctica de las Matemáticas. Barcelona, Editorial Graó, № 14, pp. 7-24.

- Socas, M. (2007). "Dificultades y errores en el aprendizaje de las Matemáticas. Análisis desde el enfoque lógico semiótico." Investigación en educación matemática: comunicaciones de los grupos de investigación del XI Simposio de la SEIEM, celebrado en La Laguna del 4 al 7 de septiembre. 